\title{
Technological Optimization of Positioning Prismatic-Body Details in Holding Devices
}

\author{
Angel Lengerov \\ Technical University - Sofia, Plovdiv \\ Branch \\ Plovdiv, Bulgaria \\ anlen@tu-plovdiv.bg
}

\begin{abstract}
The aim of the present work is to propose and substantiate a scheme of a device for positioning prismaticbody details on two holes with parallel axes and a plane, perpendicular to them. This will help to achieve high accuracy of linear and angular orientation of the workpiece. Thus the requirements for the accuracy of the center-tocenter distance of the base holes will be reduced and the process of assembling the workpiece to the grip pawls will be simplified.
\end{abstract}

Keywords - accuracy, optimization, prismatic-body details,

\section{INTRODUCTION}

The process of prismatic-body details positioning, based on two holes with parallel axes and a plane perpendicular to them, is performed on support plates with different combinations of mounting pawls: a cylindrical and a sheared pawl; two cylindrical pawls, one of which may be of reduced diameter relative to the base hole; two conical, sinking pawls; two elastic grip pawls [1,2] and others. The most common and most precise in terms of accuracy of orientation of the workpiece is the first of these solutions. The main disadvantage of positioning by means of a cylindrical and a sheared pawl is the need for achieving high accuracy of the center-to-center distance between the base holes of the workpiece during their prefabrication. This makes the technological process more expensive and is not justified in cases when the base openings are not used in mounting and assembling, but have a purely technological purpose. In addition, the operation of these devices reveals $[3,4]$ some difficulties in assembling the workpiece on the pawls.

The aim of the present work is to propose and substantiate a scheme of a device for positioning of prismatic-body details on two holes with parallel axes and a plane perpendicular to them, which would allow to achieve the highest possible accuracy of linear and angular orientation of the workpiece with reduced requirements to the accuracy of the center-to-center distance of the base holes and greater ease when assembling the workpiece to the pawls in a positioning process.

\section{METHODOLOGY}

The construction and the principle of operation of the proposed device are clarified in Fig.1. The workpiece 1 is mounted on the support plates 2 on the base surface. The pawls 3 are cylindrical. This is determined by the presence of a tolerance $T_{L}$ at a distance $L$ between the axes of the base holes, as a result of which, when positioning a batch of workpieces, the position of one of the pawls (Fig. $1 \mathrm{~b}$ ) is shared between two boundary positions. The diagram shows that the area enclosed by the intersection of the two boundary circumferences belongs to all workpieces in the batch. The diameter of the right-hand pawl must fall into this area and its tolerance must be equal to $T_{\mathrm{L}}$.

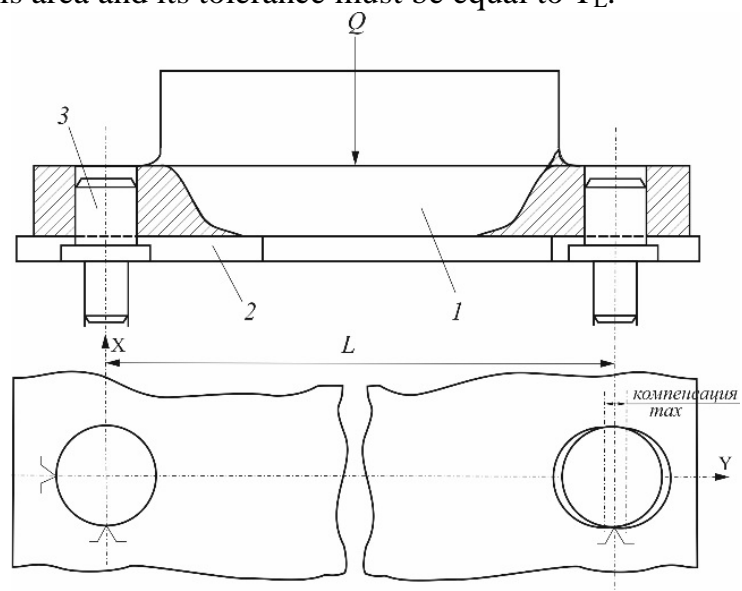

Fig.1. Model design of the device

The linear movement of the detail in the direction of Ox and Oy axes is limited within the clearance between the fixed pawl and the base hole, assembled with it. In this respect, the proposed scheme is completely equivalent to the scheme of positioning on a cylindrical and a sheared pawl. 
The limiting values for the displacement of the workpiece from the middle position are:

$$
\begin{aligned}
& J_{3 \min 1}=\frac{J_{\min 1}}{2} \\
& J_{3 \max 1}=\frac{J_{\min 1}+T_{1}+T_{1}^{\prime}+T_{w 1}}{2}
\end{aligned}
$$

where $J_{3 \min 1}$ and $J_{3 \max 1}$ are the limiting operating radial clearances;

$J_{\min 1}$ - the minimum guaranteed clearance;

$T_{1}$ - the tolerance of the base opening;

$T_{1}^{\prime}$ - the tolerance of the diameter of the pawl;

$T_{w 1}$ - the tolerance for the wear of the pawl.

The role of the movable pawl, similar to the role of the sheared one, is to limit the rotation of the detail around the axis of the fixed pawl. The largest angle of rotation $\alpha$ (Fig. 2) from the middle position can be determined by the formula

$\sin \alpha \approx \frac{J_{3 \max 1}+J_{3 \max 2}+\frac{J_{3 \max 3}}{\cos \beta}+\frac{T_{L}}{2} \sin \beta+f_{y}}{L}$,

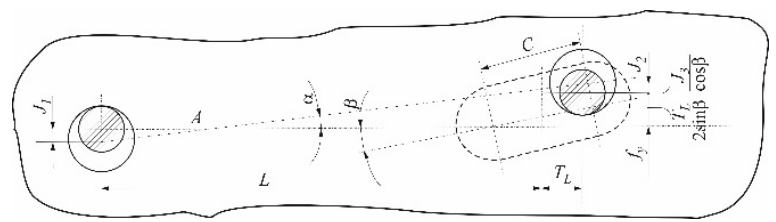

Fig. 2. Scheme for determining the error of the workpiece rotation

where $J_{3 \max 2}$ is the maximum operating radial clearance between the movable pawl and the base hole;

$J_{3 \max 3}$ - the maximum operating radial clearance between the movable pawl and the guide channel;

$\beta$ - the angle between the direction of the center-tocenter distance and the axis of the channel, obtained as a result of inaccuracy in its fabrication;

$f_{y}$ - the linear inaccuracy in the channel fabrication, representing the distance between its middle part and the direction of the center-to-center distance.

The clearance $J_{3 \max 2}$ is analogous to $J_{3 \max 1}$ and depends on the same elements as the ones, specified in (1), but taken for the connection pair movable pawl - base hole. The maximum displacement of the movable pawl in the guide channel is determined by the expression:

$$
J_{3 \max 1}=\frac{J_{\min 3}+T_{3}+T_{3}^{\prime}+T_{w 3}+T_{w 3}^{\prime}}{2}
$$

where $J_{\min 3}$ is the minimum guaranteed clearance;

$T_{3}, T_{w 3}$ are the tolerances for fabrication and wear of the pawl;

$T_{3}^{\prime}, T_{w 3}^{\prime}$ - the tolerances for fabrication and wear of the channel.

In case of positioning on a cylindrical and a sheared pawl, the maximum error of rotation of the detail, determined by the rotational angle $\alpha$, is:

$$
\sin \alpha=\frac{J_{3 \max 1}+J_{3 \max 2}+f_{y}}{L},
$$

In the above dependence, the clearances $J_{3 \max 1}$ and $J_{3 \max 2}$ completely cover by meaning the notation in (2), while $f_{y}$ is an analogous inaccuracy in the position of the sheared pawl.

The analysis of the errors in the angular position of the workpiece for the two positioning schemes, expressed by (2) and (4), shows that in the proposed scheme the error is greater, because in (3) the expressions $\frac{J_{3 \max 3}}{\cos \beta}$ and $\frac{T_{L}}{2} \sin \beta$ are added. This conclusion is still not sufficient grounds to reject the scheme, however. The performed calculations show that in practice the difference between the errors in the two schemes is small and acceptable for the production conditions.

The quantity $\frac{T_{L}}{2} \sin \beta$ can be neglected as infinitesimal, since the tolerance $T_{L}$ is a small number and the angle $\beta$ is also small $\left(\beta<1^{\circ}\right)$. As it can be seen from (3), $J_{3 \max 3}$ depends on the accuracy of manufacturing both the pawl and the channel, as well as on their wear. The capabilities of the modern tool shops allow for making both elements with high accuracy and a small minimum guaranteed clearance. Moreover, this accuracy should be considered in relation to the required accuracy of positioning the detail. The wear of both the pawl and the channel is very small due to the fact that the relative displacement path is small and the two details are in constant contact (unlike the case with the connection pair pawl-base hole).

The influence of the tolerance $T_{L}$ of the center-tocenter distance on the accuracy of the angular orientation remains implicit, as it manifests itself in different directions. When positioning on a cylindrical and a sheared pawl, the increase of $T_{L}$ leads to inadmissible sharpening of the sheared pawl, and after a certain value of $T_{L}$ - to elimination of the cylindrical band. In these cases the scheme is not applicable, and the only way out is to increase the dimensional accuracy $L$ of the 
workpieces. The inaccuracy of the device additionally interferes with this scheme.

The proposed positioning scheme with two cylindrical pawls, one of which movable, allows for full compensation of the tolerance of the size $L$ both for the workpiece and for the device, without limiting their nominal practical values. This is achieved at the expense of the free run of the movable pawl. The length of the channel (Fig. 2) when ignoring its angular error is determined by the condition:

$$
C \geq T_{L}+T_{L}^{\prime}-J_{\min 1}
$$

(5)

Two operational advantages of the proposed scheme follow from the above, compared to the case of positioning on a cylindrical and a sheared pawl - greater ease of assembly of the workpiece to the pawls and applicability in large-sized details processing.

\section{CONCLUSIONS}

1. The proposed scheme for body details positioning on two cylindrical pawls, one of which movable, provides high accuracy of angular positioning of details, comparable to the accuracy of positioning on a cylindrical and a sheared pawl. This is achieved by practically unlimited deviations in the center-to-center distance of the base openings.

2. The technological process of pretreatment of the base openings is simplified and the prime cost of the product is reduced, while the technological possibilities of the scheme expand toward large-sized details positioning.

3. In terms of details linear orientation accuracy both schemes are equal.

4. The introduction of a movable pawl determines greater ease when assembling the workpiece to the pawls in a positioning process.

\section{REFERENCES}

[1] G. Jordanov, Technological equipment, Sofia, 2014, (In Bulgarian).

[2] I. Zamfirov, Technological equipment, Ruse, 1994, (In Bulgarian).

[3] H. Metev, M. Vlahova, Modeling of the process selection of clamping devices in the design of fixtures for the locating of workpieces during machining. International scientific conference UNITECH'20, Gabrovo, vol.2, p. 33-38, 2020, (In Bulgarian).

[4] Z. Zlatanov, R. Raychev, Ch. Pashinski, Matrix examination of static undefined planar frame under static load", International Scientific Conference "TechSys 2018" - ENGINEERING, TECHNOLOGIES AND SYSTEMS, Technical University of Sofia, Plovdiv Branch, 17-19 May 2018, Plovdiv, Bulgaria. 